\title{
Efeito de um Programa de Condicionamento Físico no Broncoespasmo Induzido Pelo Exercício em Mulheres Obesas
}

\author{
Effect of a Physical Fitness Program on the Exercise-Induced \\ Bronchospasm in Obese Women
}

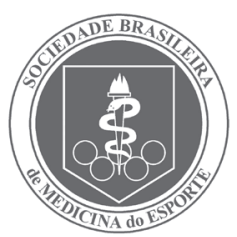

Artigo Original
Simone Padilha Cavalcante Matteoni

Carlos Roberto Bueno Júnior' Luzimar Raimundo Teixeira'

1. Escola de Educação Física e Esporte, Universidade de São Paulo, São Paulo, SP.

\section{Endereço para correspondência:}

Simone Padilha Cavalcante

Matteoni

Grupo de Estudo de Atividade Motora Adaptada (Geama) Esporte da Escola de Educação Física e Esporte da Universidade de São Paulo

Av. Prof. Mello Moraes, 65 05508-030 - São Paulo, SP E-mail: efadaptada@terra.com.br

Submetido em 16/06/2008 Versão final recebida em 02/11/2008 Aceito em 07/12/2008

\begin{abstract}
RESUMO
O broncoespasmo induzido pelo exercício físico (BIE) é uma síndrome clínica caracterizada pelo estreitamento brônquico e ocorre mais frequentemente em indivíduos obesos. Objetivo: Avaliar o possível efeito de um programa de condicionamento físico em relação ao BIE em mulheres obesas. Métodos: Trinta e duas mulheres foram divididas em três grupos: grupo A (obesidade ou sobrepeso + treinamento físico), grupo B (obesidade ou sobrepeso) e grupo C (controle). Foi aplicado um teste de caminhada de seis minutos antes e após um período de 12 semanas. O BIE foi verificado por meio de um monitor de pico de fluxo expiratório após a aplicação do teste de caminhada. Durante essas 12 semanas, para o grupo A um programa de atividade física foi conduzido três vezes por semana e cada sessão de 60 minutos foi composta por 10 minutos de alongamento, seguidos por 30 de exercícios aeróbios (50\% da frequência cardíaca de reserva como intensidade), 15 de exercícios de força de baixa intensidade e cinco de relaxamento e alongamento. Resultados: O programa de treinamento físico causou aumento significativo no pico de fluxo expiratório mínimo após o teste de esforço (pré: $379 \pm 161 /$ min; pós: $405 \pm 121 /$ min; $p \leq 0,05$ ) e reduziu em 50\% (33\% para 17\%) a proporção de mulheres que apresentou BIE $(p \leq 0,05)$. Conclusão: O programa de condicionamento físico de 12 semanas foi capaz de melhorar a função respiratória de mulheres obesas.
\end{abstract}

Palavras-chave: obesidade, atividade física, BIE.

\section{ABSTRACT}

Exercise-induced bronchospasm (EIB) is a syndrome characterized by reduced bronchial lumen and happens more often in obese subjects. Objective: To evaluate the possible effect of an exercise training program on EIB in obese women. Methods: 32 women were divided in three groups: group A (obesity + exercise training), group B (obesity) and group C (control). A six-minute walking test was applied before and after 12 weeks. ElB was measured through a monitor of peak of expiratory flow used after the application of the walking test. During the 12 weeks, an exercise training program was delivered to group A, three times a week, 60 minutes a day. Each exercise session consisted of 10 minutes of stretching exercises, followed by 30 minutes of aerobic exercise (50\% of reserve heart rate as intensity), $15^{\prime}$ of low-intensity strength training and $5^{\prime}$ of stretching and relaxing exercises. Results: Exercise training significantly increased minimum peak of expiratory flow after the walking test (before: $379 \pm 16 \mathrm{l} / \mathrm{min}$; after: $405 \pm 12 \mathrm{l} / \mathrm{min} ; \mathrm{p} \leq 0.05$ ) and reduced in $50 \%$ (33\% to 17\%) the proportion of women who presented EIB ( $p \leq 0.05)$. Conclusion: The 12-week exercise training increased the respiratory function of obese women.

Keywords: obesity, physical activity, EIB.

\section{INTRODUÇÃO}

No mundo e no Brasil, a obesidade tem sido preocupante por desencadear doenças cardiovasculares, hipertensão arterial, diabetes mellitus tipo II, artrite, tumores, desvios posturais, depressão e doenças respiratórias ${ }^{(1)}$. Atualmente, a obesidade é considerada pela Organização Mundial de Saúde (OMS) um problema de saúde pública ${ }^{(2)}$. Em países em desenvolvimento, a obesidade na população adulta vem aumentando de modo alarmante.

Das doenças respiratórias, a asma e a rinite alérgica são as que mais atingem a população mundial. A associação de duas ou mais doenças tem consequências graves, principalmente da obesidade com as doenças respiratórias. Além disso, só recentemente, com o aumento suficiente da prevalência da obesidade e possibilidades de estudos em grandes amostras populacionais, conseguiu-se determinar combinações estatisticamente significantes entre elas ${ }^{(3)}$. Essa associação ocorre porque a obesidade pode afetar o tórax, o diafragma e os músculos abdominais, determinando alterações na função respiratória ${ }^{(4)}$.

Por outro lado, inúmeros estudos têm demonstrado que a prática regular de atividades físicas promove muitos benefícios, tanto para o bem-estar do indivíduo, como a melhora clínica da obesidade e dos distúrbios respiratórios, apesar de os indivíduos obesos demonstrarem 
baixa condição física e maior probabilidade de ocorrência de broncoespasmo induzido pelo exercício (BIE) ${ }^{(8-14)}$. Dessa forma, torna-se importante verificar a ocorrência do broncoespasmo e os possíveis efeitos de um programa de condicionamento físico na diminuição do BIE em mulheres obesas.

O BIE é uma síndrome clínica caracterizada pelo estreitamento brônquico, ocorrendo obstrução transitória do fluxo aéreo. Esse estreitamento brônquico é desencadeado pela hiperventilação em decorrência do esforço físico. Ele ocorre em 90\% dos asmáticos, em 40\% dos atópicos (pessoas com rinite alérgica, eczema, urticária e outras manifestações de fundo alérgico) e há evidências de sobrevir em 10\% dos indivíduos que não apresentam os sintomas das doenças alérgicas e em atletas ${ }^{(15-17)}$. O BIE geralmente ocorre diante de um exercício com duração mínima de cerca de seis a oito minutos e intensidade entre 70 e 90\% do volume máximo de oxigênio previsto. Entre cinco e 15 minutos após cessar o exercício, havendo queda do pico de fluxo expiratório forçado (PFE) de 10\%, confirma-se o diagnóstico do BIE ${ }^{(14)}$. Essa situação tende a se reverter em torno de 30 a 60 minutos após o término do exercício com ou sem o uso de medicamento, podendo ocorrer resposta tardia em torno de quatro a 10 horas após o primeiro broncoespasmo ${ }^{(14,18)}$. Os mecanismos do BIE não estão totalmente esclarecidos, porém, há fortes evidências de que os principais motivos estão relacionados com o esfriamento e ressecamento das vias aéreas durante a atividade ${ }^{(14)}$. Os sintomas surgem devido à inflamação e ao estreitamento dos brônquios e os principais são: tosse, falta de ar, cansaço, sensação de "aperto no peito", chiado, secreção e dores de estômago ${ }^{(18,19)}$. Ressalta-se a escassez de trabalhos na literatura internacional e a falta de estudos na literatura nacional sobre as relações entre limitações respiratórias e obesidade em mulheres. Portanto, nossos objetivos foram avaliar os possíveis efeitos, pré e pós-aplicação de um programa de condicionamento físico, sobre os valores do pico de fluxo expiratório em mulheres obesas e a ocorrência do BIE.

\section{MÉTODOS}

O presente estudo foi aprovado pelo Comitê de Ética em Pesquisa da Escola de Educação Física e Esporte da Universidade de São Paulo, protocolo no 2006/07, obedecendo às diretrizes que regulamentam pesquisas envolvendo seres humanos, que constam da Resolução do Conselho Nacional de Saúde no 196/96. Todas as voluntárias que participaram da pesquisa assinaram o termo de consentimento livre e esclarecido.

Para este estudo foram selecionadas 32 mulheres com idade acima de 25 anos, sem cardiopatias e sedentárias. Foram incluídas as mulheres com sobrepeso (IMC $\geq 25$ a $<30$ ) e obesas de grau I, II e III (IMC $\geq 30$ a < 44,9), conforme as recomendações da Organização Mundial da Saúde ${ }^{(20)}$.

A amostra foi dividida em três grupos ( $A, B$ e $C$ ). O grupo A, composto de 18 mulheres obesas e sobrepeso, foi submetido ao programa de condicionamento físico de 12 semanas do curso de Educação Física do Serviço de Cultura e Extensão da Escola de Educação Física e Esporte da Universidade de São Paulo (EEFEUSP). Formaram o grupo B oito mulheres obesas e com sobrepeso que não foram sorteadas para participar do programa de condicionamento físico; o grupo C foi composto por seis mulheres eutróficas que eram funcionárias da área de limpeza da EEFEUSP.

Foram excluídas da amostra mulheres com cardiomiopatias, com idade inferior a 25 anos, que faziam uso de qualquer medicamento para doenças respiratórias e que participavam de qualquer programa de atividade física regular de seis meses antes do início do estudo até seu término. A única exceção são as mulheres do grupo A, que participaram apenas do programa descrito abaixo. O controle dessas variáveis foi realizado regularmente por meio de questionamentos às voluntárias. Além disso, todos os grupos foram avaliados na mesma época do ano, evitando o efeito de variáveis intervenientes.

O programa de condicionamento físico teve a duração de 12 semanas e havia três aulas por semana. Cada aula consistia de 10 minutos de alongamento, seguidos por 30 de exercícios aeróbios (50\% da frequência cardíaca de reserva como intensidade), 15 de exercícios de força de baixa intensidade e cinco de relaxamento e alongamento. Foi considerada requisito, para as mulheres do grupo A continuarem no estudo, a frequência mínima de $70 \%$ no programa de condicionamento físico; não foram permitidas mais do que quatro faltas consecutivas.

A medida da massa corporal foi obtida por meio de uma balança mecânica da marca Filizola, com capacidade para 150kg e sensibilidade de 100g, de acordo com a metodologia descrita por Lohman et al. ${ }^{(21)}$. A avaliação da massa corporal ocorreu no primeiro momento da triagem das voluntárias e no momento prévio de cada teste.

A estatura foi obtida por estadiômetro com haste móvel e com escala de precisão de $0,1 \mathrm{~cm}$, de acordo com metodologia já descrita ${ }^{(21)}$. A medida da estatura foi realizada no primeiro momento da triagem das voluntárias e após o período experimental.

O cálculo do índice de massa corporal (IMC) foi realizado no primeiro momento da triagem das voluntárias e após o período experimental. Foi calculado por meio da relação matemática entre a massa corporal e a estatura.

A obesidade total foi avaliada por meio do IMC e classificada como: sobrepeso para IMC $\geq 25 \mathrm{~kg} / \mathrm{m}^{2}$ e $<30 \mathrm{~kg} / \mathrm{m}^{2}$; e obesidade para a medida $\geq 30 \mathrm{~kg} / \mathrm{m}^{2}$ (22)

O monitor de pico de fluxo expiratório (PFE) é um aparelho portátil, modelo Mini-Wright, da marca Clement Clarke, fabricado na Inglaterra. Esse monitor de PFE serve para medir a velocidade a que o ar é expelido dos pulmões. Quando ocorre o BIE, os brônquios encontram-se estreitados, dificultando a passagem do ar; o monitor de PFE é capaz de indicar o grau de estreitamento.

As medidas do fluxo expiratório foram efetuadas, sob supervisão de observador único, obedecendo aos critérios de manuseio. Após três medidas, o maior valor obtido foi anotado e comparado com a tabela de valores previstos da American Thoracic Society (ATS) ${ }^{(18)}$. Essa avaliação foi realizada antes do teste de caminhada e cinco, 10, 15 e 20 minutos após seu término, tanto antes como após o período experimental.

Foi considerada como desencadeamento do BIE a queda igual ou superior a $10 \%$ em relação aos valores obtidos no repouso préexercício, até 20 minutos após o término do exercício, conforme a determinação da ATS ${ }^{(18)}$.

Determinadas condições de temperatura e umidade relativa do ar podem favorecer o aparecimento de broncoespasmo induzido pelo exercício. Para melhor controle, o teste foi realizado na pista de atletismo da EEFEUSP com a temperatura e a umidade relativa do ar respectivamente, $20-25^{\circ} \mathrm{C}$ e $45-55 \%$, verificadas por meio do aparelho manual termo-higrômetro analógico, marca TFA, de origem alemã.

O teste de caminhada foi realizado de acordo com as diretrizes estabelecidas pela ATS. Os equipamentos necessários para a realização do teste foram: cronômetro (Sport Timer ${ }^{\circ}$ ), trena, monitor de frequência cardíaca, monitor de pico de fluxo expiratório e o termo-higrômetro.

No teste de caminhada (pré e pós-período experimental), as participantes da pesquisa caminharam na pista, com a maior velocidade possível; foram orientadas a interromper o teste caso sentissem dores em membros inferiores, taquicardia ou apresentassem qualquer outro sintoma de desconforto. O teste de caminhada teve a duração de seis minutos. O monitoramento da frequência cardíaca (FC) foi realizado por um sensor de batimentos cardíacos da marca Polar. 
É importante ressaltar que obesos apresentam limitações em atividades aeróbias e alguns não alcançam a frequência cardíaca máxima. Nesses casos não ocorre o BIE e/ou sua magnitude é alterada.

Para o teste, as mulheres que estivessem em uso de medicação broncodilatadora deveriam suspendê-la com antecedência de oito horas, desde que houvesse autorização por escrito do próprio médico da voluntária; todavia, nenhuma fazia uso desse tipo de medicamento.

As mulheres foram submetidas a avaliações antropométricas, PFE e um período de aquecimento de três minutos antes do início do teste de caminhada.

Após o teste, foram mensurados a frequência cardíaca ( $F C$ ), o pico de fluxo expiratório (PFE) pós-teste e o PFE a cada cinco minutos até completar 20 minutos.

Em relação à análise estatística, utilizou-se o programa Statistica, versão 8; os dados estão expressos como média \pm erro padrão da média (EPM).

Para as variáveis idade, estatura e pico de fluxo expiratório previsto, os dados dos três grupos foram comparados por meio de análise de variância (ANOVA) de uma via.

Em relação às variáveis massa corporal, índice de massa corporal, frequência cardíaca de repouso, distância e frequência cardíaca obtidas no teste, e picos de fluxo expiratório de repouso e mínimo após o teste, os três grupos foram comparados, nos momentos pré e pós-período experimental, utilizando-se ANOVA de duas vias para medidas repetidas. Quando houve a necessidade de um teste post-hoc, utilizou-se o de Duncan.

Por fim, o teste não paramétrico e pareado de Wilcoxon foi utilizado para comparar a proporção de indivíduos que apresentaram broncoespasmo induzido pelo exercício pré e pós-período experimental.

\section{RESULTADOS}

Neste estudo, as características gerais dos grupos A (obesidade e condicionamento físico), B (obesidade) e C (controle) foram semelhantes. Na tabela 1, na qual são mostrados os dados de idade, estatura e pico de fluxo expiratório previsto. Comparando-se os grupos A, B e C nessa avaliação inicial, nota-se que não houve diferença significativa entre eles ( $p>0,05$ para todas as variáveis). Vale ressaltar os grupos A e B são compostos por mulheres com obesidade e sobrepeso - referimos como "obesidade" apenas para facilitar a nomeação dos grupos.

Tabela 1. Pico de fluxo expiratório previsto e seus determinantes (idade e estatura)

\begin{tabular}{l|c|c|c}
\hline & $\begin{array}{c}\text { Grupo A } \\
\mathbf{N}=\mathbf{1 8}\end{array}$ & $\begin{array}{c}\text { Grupo B } \\
\mathbf{N = \mathbf { 8 }}\end{array}$ & $\begin{array}{c}\text { Grupo C } \\
\mathbf{N}=\mathbf{6}\end{array}$ \\
\hline Idade (anos) & $46,6 \pm 2,0$ & $44,4 \pm 3,0$ & $38,5 \pm 3,4$ \\
\hline Estatura (cm) & $157,1 \pm 1,2$ & $160,0 \pm 2,3$ & $160,3 \pm 2,9$ \\
\hline PFE previsto (I/min) & $457,8 \pm 3,1$ & $463,0 \pm 4,7$ & $468,7 \pm 5,0$ \\
\hline \multicolumn{2}{|l|}{ Os dados estão expressos como média \pm EPM. PFE: pico de fluxo expiratório. }
\end{tabular}

Os dados estão expressos como média \pm EPM. PFE: pico de fluxo expiratório.
Na tabela 2 pode ser observado que tanto o grupo A (obesidade e condicionamento físico) como o grupo B (obesidade) apresentaram valores de massa corporal e índice de massa corporal significativamente $(p \leq 0,05)$ maiores do que os do grupo $C$ (controle). O programa de condicionamento físico, no entanto, não foi capaz de reduzir a massa corporal e o índice de massa corporal.

Tabela 2. Massa corporal, índice de massa corporal e frequência cardíaca de repouso

\begin{tabular}{l|c|c|c|c|c|c}
\hline & \multicolumn{2}{|c|}{$\begin{array}{c}\text { Grupo A } \\
\mathrm{N}=18\end{array}$} & \multicolumn{2}{c|}{$\begin{array}{c}\text { Grupo B } \\
\mathbf{N}=\mathbf{8}\end{array}$} & \multicolumn{2}{c}{$\begin{array}{c}\text { Grupo C } \\
\mathbf{N}=6\end{array}$} \\
\cline { 2 - 7 } & Pré & Pós & Pré & Pós & Pré & Pós \\
\hline Massa corporal $(\mathrm{kg})$ & $77,7 \pm 2,5^{*}$ & $77,3 \pm 2,5^{*}$ & $83,4 \pm 4,0^{\$}$ & $84,1 \pm 4,0^{\$}$ & $62,1 \pm 2,1$ & $62,1 \pm 2,0$ \\
\hline IMC $\left(\mathrm{kg} / \mathrm{m}^{2}\right)$ & $31,5 \pm 0,9^{*}$ & $31,3 \pm 0,8^{*}$ & $32,6 \pm 1,4^{\$}$ & $32,8 \pm 1,3^{\$}$ & $24,2 \pm 0,3$ & $24,2 \pm 0,3$ \\
\hline FCrepouso (bpm) & $82,3 \pm 1,7$ & $80,2 \pm 1,3$ & $85,9 \pm 1,9$ & $85,3 \pm 2,0$ & $78,3 \pm 1,7$ & $76,7 \pm 2,1^{\#}$ \\
\hline
\end{tabular}

*: $p \leq 0.05$ vs. Grupo B (Pré e Pós) e Grupo C (Pré e Pós)

\$: $p \leq 0.05$ vs. Grupo C (Pré e Pós)

$\#: p \leq 0.05$ vs. Grupo B (Pré e Pós)

Os dados estão expressos como média \pm EPM. IMC: índice de massa corporal. FC: frequência cardíaca.

Já a distância percorrida foi estatisticamente maior ( $p \leq 0,05)$ no grupo C (controle) do que nos grupos A (obesidade e condicionamento físico) e B (obesidade) (tabela 3). Assim como em relação à massa corporal e ao índice de massa corporal, o programa de condicionamento físico não foi capaz de alterar os resultados dessa variável.

O pico de fluxo expiratório de repouso apresentou-se estatisticamente reduzido $(p \leq 0,05)$ no grupo $C$ (controle) em relação aos grupos A (obesidade e condicionamento físico) e B (obesidade) (figura 1). O programa de condicionamento físico não foi capaz de alterar o pico de fluxo expiratório de repouso, mas aumentou significantemente $(p \leq 0,05)$ o pico de fluxo expiratório mínimo pós-teste, pois o grupo A (obesidade e condicionamento físico) após o condicionamento físico apresentou valores superiores aos encontrados antes do programa (figura 2).

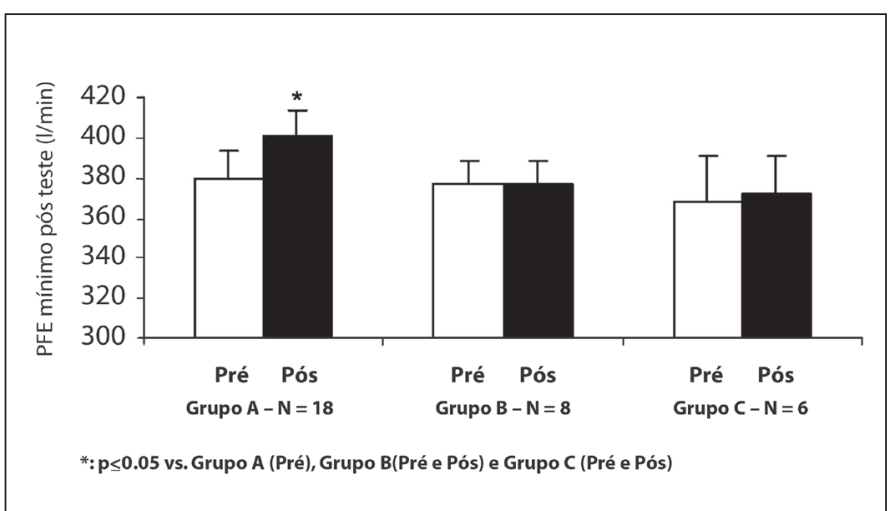

Figura 1. Pico de fluxo expiratório de repouso

Os dados estão expressos como média \pm EPM. PFE: pico de fluxo expiratório.

Tabela 3. Distância percorrida e frequência cardíaca obtidas no teste de caminhada

\begin{tabular}{|c|c|c|c|c|c|c|}
\hline & \multicolumn{2}{|c|}{$\begin{array}{c}\text { Grupo A } \\
N=18\end{array}$} & \multicolumn{2}{|c|}{$\begin{array}{c}\text { Grupo B } \\
N=8\end{array}$} & \multicolumn{2}{|c|}{$\begin{array}{c}\text { Grupo C } \\
N=6\end{array}$} \\
\hline & Pré & Pós & Pré & Pós & Pré & Pós \\
\hline Distância $_{\text {teste }}(\mathrm{m})$ & $654,2 \pm 16,6^{*}$ & $650,3 \pm 15,5^{*}$ & $590,6 \pm 33,8^{\$}$ & $583,7 \pm 35,2^{\$}$ & $755,0 \pm 21,5$ & $750 \pm 17,3$ \\
\hline $\mathrm{FC}_{\text {teste }}(\mathrm{bpm})$ & $127,1 \pm 3,1$ & $130,2 \pm 3,1$ & $129,0 \pm 3,4$ & $132 \pm 2,8$ & $143,3 \pm 7,6^{\#}$ & $138,3 \pm 4,8$ \\
\hline
\end{tabular}

*: $p \leq 0.05$ vs. Grupo B (Pré e Pós) e Grupo C (Pré e Pós)

$\$: p \leq 0.05$ vs. Grupo C (Pré e Pós)

\#: $p \leq 0.05$ vs. Grupo A (Pré e Pós)

Os dados estão expressos como média \pm EPM. FC: frequência cardíaca. 


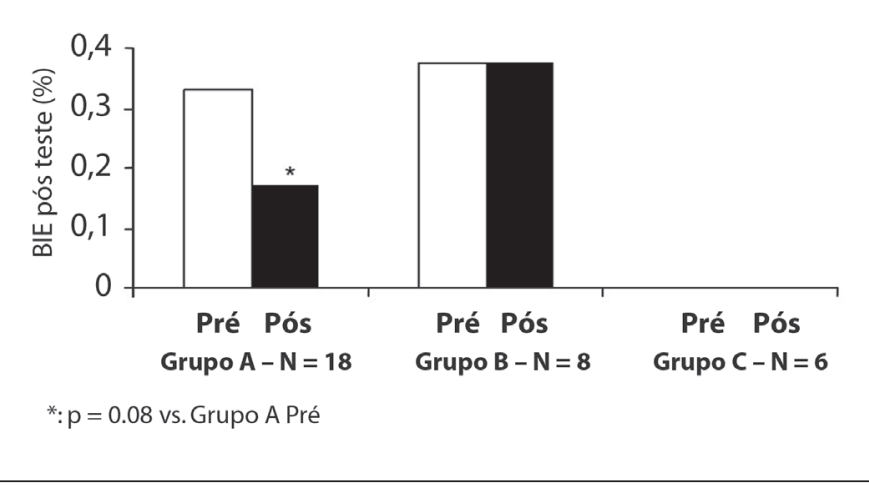

Figura 2. Pico de fluxo expiratório mínimo após o teste de caminhada Os dados estão expressos como média \pm EPM. PFE: pico de fluxo expiratório.

Corroborando o efeito do programa de condicionamento físico mostrado na figura 2, o programa também reduziu em 50\% ( $p \leq 0,05)$ a proporção de mulheres que apresentaram broncoespasmo induzido pelo exercício pós-teste (figura 3).

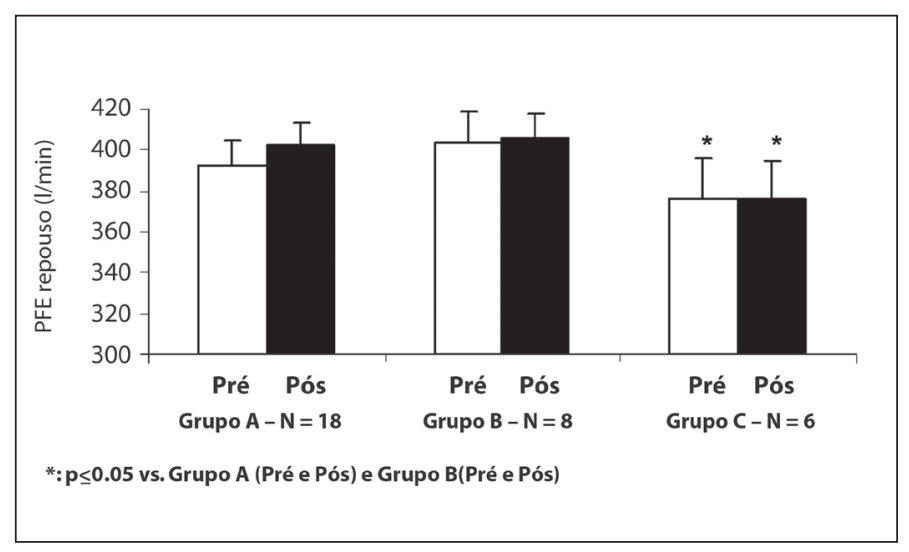

Figura 3. Proporção de broncoespasmo induzido pelo exercício Os dados estão expressos como média \pm EPM. BIE: broncoespasmo induzido pelo exercício.

\section{DISCUSSÃO}

Inicialmente, foi demonstrado que as variáveis gerais de idade, estatura e pico de fluxo expiratório previsto foram iguais nos três termos, o que mostra a homogeneidade entre os grupos.

Posteriormente, foi demonstrado que os grupos A e B, com obesidade e sobrepeso, conforme esperado, apresentaram valores maiores de massa corporal e índice de massa corporal do que o grupo controle (C). O programa de condicionamento físico não foi capaz de reduzir os valores dessas variáveis e isso pode estar relacionado com o tempo de duração do programa, que foi de apenas três meses.

Conforme previsto, os grupos com obesidade e sobrepeso (A e B) também apresentaram menor distância percorrida no teste de esforço do que o grupo controle $(C)$ e isso pode ser explicado pela menor intensidade alcançada pelas idosas, devido à menor capacidade cardiorrespiratória e/ou fraqueza na musculatura esquelética apendicular ${ }^{(23)}$. O programa de treinamento físico também não foi capaz de aumentar a distância percorrida no teste de esforço físico e isso também pode estar relacionado com o reduzido tempo de duração do programa. Além disso, o programa de atividade física pode não ter gerado redução na gordura corporal e aumento na distância percorrida, porque é muito difícil um indivíduo obeso frequentar um programa de atividade física em associação com a adoção de hábitos saudáveis, como a alimentação(24). Apesar dessas especulações, uma explicação mais plausível para a ausência de aumento na distância percorrida pelas mulheres obesas após o programa de condicionamento físico é a ocorrência do efeito teto, ou seja, para aumentar a velocidade e, consequentemente, a distância percorrida, as mulheres teriam que correr, o que não era permitido. Essa é uma limitação do estudo; no entanto, como será demonstrado abaixo, melhoras em parâmetros respiratórios comprovam a eficiência do programa de condicionamento físico.

Em seguida, foi demonstrado que o grupo controle apresentou menor pico de fluxo expiratório de repouso em relação aos grupos obesidade e sobrepeso (A e B). Esse resultado não foi esperado inicialmente, mas pode ter ocorrido devido às características do grupo, que foi composto por funcionárias da área de limpeza que, devido ao contato com aeroalérgenos ambientais, podem apresentar alterações na função pulmonar.

Já o programa de treinamento físico, apesar de não ter alterado o pico de fluxo expiratório de repouso, causou aumento significativo no pico de fluxo expiratório mínimo após o teste de esforço físico. Esse benefício está relacionado com melhora na função pulmonar e este é o primeiro estudo na literatura a mostrar o efeito de um programa de atividade física sobre o broncoespasmo induzido pelo exercício em indivíduos obesos. Essa melhora na função respiratória é extremamente relevante em pessoas obesas, que, devido à deposição de tecido adiposo no tórax e no abdômen, apresentam menor complacência pulmonar e da parede torácica, o que compromete o sistema respiratório e o transporte de gases ${ }^{(25)}$.

Corroborando essa melhora referida anteriormente, menor proporção das mulheres do grupo A (obesidade e condicionamento físico) apresentou broncoespasmo induzido pelo exercício após o programa de treinamento físico, mostrando também a eficácia do programa em relação à função pulmonar.

De acordo com nosso conhecimento, este é o primeiro estudo na literatura que buscou avaliar o efeito de um programa de atividade física em relação a um prejuízo respiratório em indivíduos obesos, no caso, o broncoespasmo induzido pelo exercício. Grande parte dos estudos que buscam investigar o efeito de sessões de exercício físico a longo prazo em algum dano respiratório estudou crianças com asma e mostrou uma série de benefícios, como melhora nas variáveis espirométricas e redução na frequência de aparecimento e na gravidade dos broncoespasmos induzidos pelo exercício ${ }^{(26-32)}$.

Conclui-se que um programa de condicionamento físico de três meses, três vezes por semana, com sessões de 60 minutos, propiciou melhora no pico de fluxo expiratório mínimo após o teste de esforço físico e diminuição na ocorrência de broncoespasmo induzido pelo exercício em mulheres obesas. Embora as mulheres estudadas continuassem sendo obesas e, algumas, portadoras do broncoespasmo induzido pelo exercício, o condicionamento físico possibilitou-Ihes executar uma atividade física com valores de pico de fluxo expiratório pós-programa mais altos, ou seja, com menor grau de broncoespasmo induzido pelo exercício. Esses resultados podem ter implicações clínicas importantes e benéficas para essas mulheres em suas atividades diárias e, principalmente, na prática de atividades físicas.

Todos os autores declararam não haver qualquer potencial conflito de interesses referente a este artigo. 


\section{REFERÊNCIAS BIBLIOGRÁFICAS}

1. Avenell A, Broom TJ, Poobalan A, Aucott L, Stearns SC, Smith WCS, et al. Systematic review of the long-term effects and economic consequences of treatments for obesity and implications for health improvement. Health Techn Assessm 2004;21:1-182.

2. Pinheiro ARO, Freitas SFT, Corso ACT. Uma abordagem epidemiológica da obesidade. Rev Nutr 2004;17:523-33.

3. Beuther DA, Weiss ST, Sutherland ER. Obesity and Asthma. Am J Respir Crit Care Med 2006;174:112-9.

4. Chinn S, Rona RJ. Can the increase in body mass index explain the rising trend in asthma in children? Thorax 2001;56:845-50

5. Camargo Junior CA, Weiss ST, Zhang S, Willett WC, Speizer FE. Prospective study of body mass index, weight change, and risk of adult-onset asthma in women. Arch Intern Med 1999;159:2582-8.

6. Del-Rio-Navarro BE, Fanghanel G, Berber A, Sanchez-Reyes L, Estrada-Reyes E, Sienra-Monge JJ. The relationship between asthma symptoms and anthropometric markers of overweight in a Hispanic population. J Investig Allergol Clin Immunol 2003;13:118-23.

7. Gold DR, Rotnitzky R, Damokosh, Al, Dockery DW, Berkey CS. Race and gender differences in respiratory illness prevalence and their relationship to environmental exposures in children aged 7 to 14 years of age. Am Rev Respir Dis 1993;148:10-8.

8. Ashutosh K, Methrotra K, Fragale J. Effects of sustained weight loss and exercise on aerobic fitness in obese women. J Sports Med Phys Fitness 1997;37:252-7.

9. Blundell JE, King NA. Physical activity and regulation of food intake: current evidence. Med Sci Sports Exerc 1999;31:573-83.

10. Costa NP, Holderer RMG. A atividade física e o broncoespasmo induzido pelo exercício. J Asthma 1989;4:182-9.

11. Denadai RC, Vítolo MR, Macedo AS, Teixeira L, Cezar C, Dâmaso AR, et al. Efeitos do exercício moderado e da orientação nutricional sobre a composição corporal de adolescentes obesos avaliados por densitrometria óssea (DEXA). Rev Bras Educ Fís Esp 1998;12;2:210-8.

12. Fagard RH. Physical activity in the prevention and treatment of hypertension in the obese. Med Sci Sports Exerc 1999;31:624-30.

13. Jakicic JM. The role of physical activity in prevention and treatment of body weigth gain in adults. J Nutr 2002;132:382-9.

14. Virant FS. Exercise-induced bronchospasm: epidemiology, pathophysiology and therapy. Med Sci Sports Exerc 1992;24:851-5.

15. Teixeira LR, Magalhães RX, Freitas SL, Villa F. Asma: da teoria à prática. In: SIMPÓSIO PAULISTA de EDUCAÇÃO FíSICA ADAPTADA, 7., São Paulo, 1998. Anais. São Paulo: CEPEUSP 1998;121-9.

16. Chinn S. Obesity and asthma: evidence for and against a causal relation. The J Asthma 2003;40:1-10.
17. Matteoni SPC, Teixeira LR. Broncoespasmo induzido pelo exercício em atletas de natação - a natação é uma das atividades menos asmagênica? Rev Cient JOPEF 2005;3:30-3.

18. ATS Statement: Guideline for the six-minute walk test. Am J Respir Crit Care Med 2002;166:111-7.

19. Durham SR. Uma via aérea: o vínculo entre rinite alérgica e asma. Adv Stud Med 2002;24:861-5.

20. World Health Organization. Obesity: preventing and managing the global epidemic. Geneva: World Health Organization, 1998.

21. Lohman T, Roche A, Martorell R. Anthropometric standardization reference manual. Champaign: Human Kinetics, 1988

22. American College of Sports Medicine. Guidelines for exercise testing and exercise prescription. $6^{\text {th }}$ ed. Williams \& Wilkins, 2000.

23. Ashutosh K, Methrotra K, Fragale J. Effects of sustained weight loss and exercise on aerobic fitness in obese women. J Sports Med Phys Fitness 1997;37:252-7.

24. Gordon PM, Heath GH, Holmes A, Christy D. The quantity and quality of physical activity among those trying to lose weight. Am J Prev Med 2000;18:83-6.

25. Nairmak A, Cherniak RM. Compliance of the respiratory system and its components health and obesity. J Appl Physiol 1960;15:377-82.

26. Sidiropoulou MP, Fotiadou EG, Tsimaras VK, Zakas AP, Angelopoulou NA. The effect of interval training in children with exercise-induced asthma competing in soccer. J Strength Cond Res 2007; 21:446-50.

27. Neder JA, Nery LE, Silva AC, Cabral AL, Fernandes AL. Short-term effects of aerobic training in the clinical management of moderate to severe asthma in children. Thorax 1999;54:202-6.

28. Araki H, Kano S, Nishima S, Ogasawara M, Matsuzaki M, Tanaka MM, et al. Effects of physical training on children with bronchial asthma. Arerugi 1991;40:205-14.

29. Varray AL, Mercier JG, Terral CM, Prefaut CG. Individualized aerobic and high intensity training for asthmatic children in an exercise readaptation program. Is training always helpful for better adaptation to exercise? Chest 1999:99:579-86

30. Olivia CK. Physical conditioning programme for children with bronchial asthma. Acta Paediatr Jpn 1990;32:173-5.

31. Tancredi G, Cerimoniale G, Lo Tesoriere A, Aragona P, Cutrera R, Ronchetti R. Measurement of the metabolic response to physical exercise in children. Influence of training and bronchospasm Pediatr Med Chir 1985;7:351-5

32. Haas F, Pasierski S, Levine N, Bishop M, Axen K, Pineda H, et al. Effect of aerobic training on forced expiratory airflow in exercising asthmatic humans. J Appl Physiol 1987; 63:1230-5. 